\title{
Multidisciplinary team working, clinical networks, and chambers; opportunities to work differently in the NHS
}

\author{
S Carter, P Garside, A Black
}

Qual Saf Health Care 2003;12(Suppl 1):i25-i28

Recently in the United Kingdom some new organisational structures for clinicians have been discussed. So far little has changed, but the intensity of interest suggests this may be an opportunity to link change in working practices with improvements in quality. Multidisciplinary team working is developing within the National Health Service (NHS) and some groups are expanding their roles across traditional institutional boundaries to form complex clinical networks. It would require little to make these functional networks autonomous from current NHS structures. Other models of working without traditional institutional boundaries have been discussed, including the formation of "chambers" for doctors and other professionals. We describe the first tentative steps of one group as an example and suggest that further experimentation with evaluation is required.

See end of article for authors' affiliations

Correspondence to: P Garside, Newhealth, 26 Milson Road, London W14 OU, UK
$T$ he context for the delivery of health care is changing. Patient expectations, targets such as reduced waiting times, and the constraints imposed by the European Working Time Directive have inspired some in the United Kingdom to consider new models of organising and "purchasing" clinical resources. The shortage of labour is probably the most critical issue facing the National Health Service (NHS), if the objectives of the NHS Plan are to be met. ${ }^{1}$ Even to achieve current aspirations-let alone entertain notions of the NHS moving to a managed care model, with the maintenance of central taxation but mixed ownership of provision-we will need new organisational vehicles for transaction and negotiation of all clinical resources. ${ }^{2}$ Motivation for organisational innovation may come from purchasers of care as much as from clinical staff and provider organisations. The key negotiators for provision of services-the newly formed primary care trusts-could elect to buy clinical services from other than the conventional hospital structures and may thus inspire changes in working patterns and organisational structures. Change in the organisation of clinical resources may be motivated if foundation hospitals are established within the NHS. The freedoms attached to foundation status may encourage providers to reshape clinical services and change contractual relationships with staff. The advent of health resource groups (HRGs) as a basis for payment for medical cases within the NHS from 1 April 2003 will mean that services must be reasonably priced. HRGs will form the tariff of prices for designated procedures and episodes of care, similar to diagnostically related groups as used in the United States and other countries. The tariff will enable a method of comparing traditional and new service models to be formed.

There are many pressures for changing the way clinicians work in the modern NHS. An increasing emphasis on team working has led to new groups of clinicians dedicated to specific areas of care. As these groups mature, they are increasingly sophisticated, with considerable experience in managing a small section of the health service. Such strong teams are likely to wish to gain more autonomy in order to improve their service quality. One model that has caused interest is the concept of doctors working in chambers in which autonomous groups of clinicians, to some extent independent from the NHS, sell their clinical services to both the private and the public sector. The notion of "doctors in chambers" has become an urban myth in the United Kingdom-much talked about but little practised; very few chambers have been established and, to our knowledge, all are based in the private sector. Although the model of selling services back to the NHS has yet to be established, it is clear that the banner of doctors in chambers covers a number of different possible models that need to be piloted and evaluated. We report one such example, explore some of the limitations already encountered, and consider the impact of new ways of working and clinical autonomy on the delivery of real gains in quality of health care.

\section{MULTIDISCIPLINARY TEAMS AND CLINICAL NETWORKS IN THE NHS}

In the past few years there has been considerable development in multidisciplinary working throughout the NHS. Whereas before individual consultants and doctors led small teams of assistants and trainees, the development of clinical governance has promoted the development of bigger teams with broader remits and less dominance of the individual. ${ }^{3}$ The concept of corporate responsibility rather than of the individual clinician's duty of care is further reinforced by the challenging constraints of the European Working Time Directive and other employment regulations.

There are many examples of multidisciplinary teams delivering specialist care-for example, managing care for people with cancer, diabetes, or those who have complex medical and social needs such as is the case with stroke rehabilitation. ${ }^{45}$ It is widely held that teams provide better 
care than individuals working in isolation. ${ }^{6-8}$ In the United Kingdom this approach to the delivery of better care has been stimulated by the demands of the NHS service frameworks for cancer. ${ }^{1910}$ From our experience the quality improvements that result from the process of effective multidisciplinary team working include:

- When patients know that they are being looked after by a team they get a sense of confidence similar to that from having a second opinion, reducing the fear that their treatment is based on the knowledge of just one clinician.

- It is easier to provide continuity of good quality care and clinical responsibility for patients with multidisciplinary team working in a world where the working week and the available qualified staff are both shrinking.

- The constant dynamic of an effective clinical team allows the embedding of clinical management protocols, otherwise often forgotten in clinical practice.

- Not only is protocol implementation enhanced but the audit of protocol design and its outcome is an active and continuous process.

- Communication between individual members of the team is enhanced by more frequent opportunities to speak directly to each other about clinical matters.

- Discussion of the care of individual patients leads to a cross fertilisation of ideas to other situations and patients, which would not be apparent in one to one clinical management.

- Sharing of knowledge becomes easier-you can learn without reading journals!

- The group can address issues of resource management in a more rounded way avoiding waste and improving chances of arguing effectively for more resource as needed.

- Team working increases the sense of partnership and provides friendship and support particularly in difficult clinical situations such as the management of clinical errors and complaints.

Despite the many advantages of multidisciplinary working, building effective teams in the NHS remains a challenge. The reasons for this include:

- Organisations, departments, and units within the NHS have traditionally secured resources through the creation of local power structures that attract money and staff. The concept of sharing responsibility may be thought to weaken this power base for resource acquisition.

- On an individual level, responsibility sharing might be seen to weaken the clinician's ability to help patients and achieve professional goals.

- There is insufficient time available outside the time devoted to clinical work to practice genuine reflective clinical management, team building, and development. Teams are often seen as time consuming and wasteful of resources, especially in the early stages.

- The NHS culture has a long way to go in encouraging staff to listen to the point of view of others while at the same time managing to balance the contributions of individual members and their professional views.

- Effective clinical leadership is underdeveloped and without it team working will fail particularly where there is a high turnover of team members. ${ }^{8} 1112$

- Defining the boundaries of the team may be difficult because of the multiple linkages between all staff within our organisations. It is easy to include too many individuals in the team without producing real improvements in clinical care, but instead wasting valuable clinical face time.

- As these multidisciplinary teams have matured, in our experience it seems that their sense of frustration with the current hierarchical structure of NHS management grows.

These difficulties, encountered in the setting up of multidisciplinary teams, are likely to apply to the development of other approaches to organising clinical resources, including chambers. Some are the universal barriers that emerge in any change process, but others can be linked to the traditional structure and culture of the NHS. ${ }^{13-16}$ Some of these barriers could be overcome if teams could see some prospect of acquiring a more autonomous status in the future.

Clinical network is a term that can be used to describe teams of clinicians from different organisations, offering a comprehensive range of services in different locations across traditional boundaries. (If effective groups of highly motivated individuals were not hemmed in by traditional boundaries they could consider offering their services to a wider range of purchasers across traditional boundaries.) As an example, clinical multidisciplinary teams from neighbouring organisations might decide to share referrals, or establish subspecialty practices to improve quality. Pressure for these linkages is happening because of new statutory limitations on the working hours of staff and the economic pressures for centralisation of specialist services on fewer sites. Such groups are also more likely to work across the boundary of primary and secondary care, incorporating the goals of each domain.

The history of development of clinical networks in the NHS is mixed. Many health authorities attempted to steer local service development by bringing clinicians together and agreeing guidelines. When forced together the tensions of loyalties to the different hospitals often outweigh any perceived advantages of the group working together. The most formalised clinical networks have been the cancer networks established by the Calman-Hine Report. ${ }^{17}$ These organisations are becoming effective but the goals are often strikingly different from the local hospital's management and attempts to instruct complex organisations on how to spend money on one issue among many seem to cause difficulties. For further development of effective networks perhaps hospitals will need to act as providers of facilities and support services while clinicians organise themselves into networks, groups, teams, or chambers that have some level of autonomy. Effective networks can perhaps develop as small businesses. Establishing a successful business requires effective leadership, a strong team approach, and the drive to grow. There are clear drivers for change in the NHS and teams are developing in multiple forms. Perhaps all that is needed is sound local leadership?

\section{CHAMBERS - A POSSIBLE MODEL FOR THE NHS}

Multidisciplinary teams and networks are developing as a result of drivers such as the NHS service frameworks. But there are fewer obvious external drivers for the development of chambers of clinical staff. Organising clinical resources into chambers would mean staff working in an organisational model similar to that traditionally used by the legal profession. ${ }^{18}$ The term chambers in the context of health care is commonly used generically to refer to alternative organisational forms for doctors to sell their services to the NHS and other payers. Cooperative might be a better term than chambers if staff remain employed in the NHS with some autonomy from the monolithic NHS structure. Such models have traditionally been perceived to involve doctors, but to be more effective they should include other groups of staff as well. The chambers of doctors that do exist are all based in 
the private sector and most are in south east England, to our knowledge.

The concept of medical chambers is only loosely defined, but there are some common underlying assumptions:

- The advantage of working in chambers is that it provides efficiencies of scale and organisation (this is usually coupled with an assumption that quality of life for members of the chambers would be improved).

- The chambers would negotiate with a variety of agencies for work.

- The individual within the chambers would be under some new form of managerial control and not that which is customary for staff employed in the NHS.

- The chambers would be organised in such a way that streamlined processes and skill utilisation would improve productivity and quality of care.

Radical changes to the way clinicians work will only happen if there are clear advantages to patient care and if clinicians see advantages both to their patients and themselves. This is not about remuneration but about quality of working lives. Just as the resources for such changes are likely to be those of time and drive rather than finances. We describe here the attitudes of a group of surgeons to the prospect of changing their work from that of the traditional NHS monolithic hierarchy to a chambers approach.

\section{CASE STUDY}

A group of 27 urological surgeons in London explored the possibilities of a new organisational arrangement early in 2002. Some useful insights emerged. Colleagues were asked to submit their existing job plans for both NHS and private work, and to file their ideal job plans. Sufficient information emerged to suggest that progress could be made towards developing working patterns to suit individual surgeons through working as a group. Some wanted more teaching while some wanted to get out of it altogether. Some wanted more complex cases while others wanted less. Special interests were reasonably spread and there were many points of agreement:

- Bigger groupings were desirable. The arrangement of nine groups of only three surgeons in each was generally thought a poor framework for organising the speciality.

- Substantial increases in quality of care and productivity would be delivered if the whole group worked together and if control of the basic resources (theatres, equipment, key staff) and agreed processes and systems (records, scheduling, appraisal, training) could be established.

- Although money will always be important, it was not the primary factor driving the decision to investigate the option of a chambers type of arrangement.

- The patient's access to and progress through the system of care could be streamlined and improved by agreeing changes in working practices.

- Avoiding the duplication of surgical services on several sites could produce significant gains in quality. However, it was also agreed that good quality urology services required an extensive network of other clinical disciplines and could not easily be performed in a stand alone diagnostic and treatment centre or small hospital.

- There was a widely shared desire to regain control of their professional lives. The need to juggle transport, home life, and work commitments, while battling with the system to get the job done, was clearly regarded as unsustainable.

Some obvious difficulties appeared early in the discussion:
- There was fear and anxiety about taking a line that might be deemed hostile either to the NHS as a whole or to particular hospitals.

- There were also anxieties around power within the group: would some colleagues become too powerful? Could it be better to be managed even by a manager rather than by one of your own?

- There was a reluctance to give up current clinical and management roles that encompassed responsibility for several specialities-the surgeons did not want to become "ghettoised" into single speciality working.

- The group regarded the training of junior surgeons as a high priority and concerns were raised that this could be adversely affected by forming a clinical autonomous group.

- Clinical information systems are too inefficient to allow easy movement of clinical care from place to place or to allow accurate tracking of the group's activity across several hospital organisations.

- Ways of dealing with pension arrangements were critical to establish an enterprise devolved from the NHS.

As a result of these discussions a smaller group of 12 urological surgeons from three trusts and four hospital sites have started working together with arrangements to cover each other's practices, agreed guidelines for referral, and developed internal subspecialist practices. The team is multidisciplinary and works across organisational boundaries and can thus be termed a clinical network. The clinical network or pre-chambers is now in a position to negotiate with primary care trusts and is already changing referral practice and surgical activity from site to site so as to optimise efficiency. Quality of care has been enhanced by the application of standard protocols and the centralisation of complex care for particular procedures to each site. The management of emergency care has been considerably enhanced because of the ability to provide a dedicated team of urologists on one site, which would have been impossible at each individual hospital. Generally the group is much more self reliant and feels more able to control its work, and thus feels more autonomous. Currently, this group has the support of the trusts that employ the surgeons even when at times the group's goals are not the same as those of the individual trusts. An example of this is when new staff are appointed. In this case, the network might like to have a loose job plan which could be portable across the institutions, but the individual trusts might worry about accountability and the best method for sharing the cost between the institutions. Further developments with a centralised referral system and initiatives to take on additional work are being planned. Interestingly, the group has expressed no wish to include their private practice work and for the moment this remains a NHS initiative. There is now a need to learn more about ways in which this group can develop a formal organisational structure that can fit into a wider family of NHS organisations.

\section{DISCUSSION}

A significant barrier to achievement of improved care in the NHS including those outlined in the NHS is the shortage of clinical staff. This is a possible stimulus for reform of the organisation of care so that clinical staff are organised in ways better able to meet the needs of today's patients. The NHS should harness this energy, and encourage such initiatives so that it can find new ways through which effective and functional teams can deliver health care. This is a unique opportunity to renegotiate the organisation and deployment of the most valuable resource: highly trained 


\section{Key messages}

- Multidisciplinary team working provides many opportunities to improve the quality of health care.

- Clinical networks are developing across traditional NHS boundaries.

- The concept of chambers for NHS staff is much talked about but largely an untried idea.

- New organisational structures are emerging to challenge the traditional model of employment in the NHS and should be encouraged and evaluated.

- More research is needed on the outcomes of multidisciplinary team working, new ways of organising healthcare staff, and the effect of both on the quality of care.

- Investigate many different models for employing groups of staff which exist both in other industries and health systems, to determine which would be most suitable for the NHS, and which offer the greatest opportunity for better safer health care.

health care professionals. It does not have to be chambers, but a new organisational model or models for the delivery of health care are needed to modernise the way in which we use teams and staff. The development of strong clinical teamsfor example, for cancer care-is an important first step and has allowed much local experimentation. As clinical teams mature and more frequently work across boundaries some may want more autonomy. There is an inherent assumption that modernisation of systems and processes bring improvements in quality although it is necessary to test this hypothesis carefully. It is likely that effective and functioning teams do provide better care. ${ }^{14}$

In our view the way we currently organise clinical care does not suit the delivery of modern health care. For real improvements in the quality of care significant changes will need to be made to the way in which clinical resources are organised. There are many drivers for change; important changes have already happened, and our experience of one group of surgeons has found a new way of working that provides a better quality of working life. Of course, there are many obstacles-for example, related to remuneration-but change seems likely. It is crucial, however, that we do not make assumptions about the effects of such changes on the quality or safety of care but that new approaches to constructing working patterns and relationships are each evaluated rigorously as they are developed. In our view emerging organisational forms within the NHS should be encouraged, taken several steps further, and evaluated to see if they realise the potential to improve quality of care to patients.
Perhaps the NHS should harness this energy, and encourage such initiatives. If we can find new ways through which effective and functional teams can deliver health care then this could be beneficial for patients and staff. We should not allow existing, possibly outdated, structures that may be nearing their use by date to dictate the way that we organise our work, if we can organise clinical professionals in a way that provides better care. With this in mind, we need to explore ways by which this apparent growing desire for ways of working and clinical autonomy can deliver real gains in quality of health care.

\section{Authors' affiliations}

S Carter, Hammersmith Hospitals Trust and Imperial College School of Medicine, London, UK

P Garside, Judge Institute of Management, University of Cambridge, Cambridge, UK

A Black, Durrow, PO Box 22, Machlynlleth, Wales SY20 8WQ

Declaration of interest: $S C$ and $A B$ led the conversations with the group of urological surgeons regarding the formation of chambers. Both PG and $A B$ are keen to find new groups of clinicians who wish to pursue alternative working arrangements and thereby in the future might benefit financially in advising them.

\section{REFERENCES}

1 Department of Health. The NHS plan: a plan for investment, a plan for reform. London: HMSO, 2000.

2 Alan Milburn. Speech by Chief Secretary to the Treasury, Alan Milburn, at the launch of the IPPR commission into public/private partnerships, 20 Sept 1999. www.hm-treasury.gov.uk/newsroom_and_speeches/speeches (accessed 16 Sep 2003)

3 Department of Health. Quality in the new NHS: a first class service. London: Department of Health, 1998.

4 Laffel LM, Vangsness L, Connell A, et al. Impact of ambulatory, family focused teamwork intervention on glycemic control in youth with type I diabetes. J Pediatr 2003;142:409-16

5 Indredavik B, Bakke F, Slordahl SA, et al. Treatment in combined acute and rehabilitation stroke unit: which aspects are most important? Stroke 1999:30:917-23.

6 Borrill C, West $M$, Shapiro $D$, et al. Team working and effectiveness in health care. British Journal of Health Care Management 2000;8:364-70.

7 Carter A, West M. Sharing the burden: team work in health care setting. In: Firth-Cozens J, Payne R, eds. Stress in health professionals. Chichester: John Wiley, 1999:191-202.

8 Firth-Cozens J. Cultures for improving patient safety through learning: the role of teamwork. Qual Health Care 2000;10(Suppl ii): 26-31.

9 Department of Health. National service framework for diabetes standards. London: Department of Health, 2001.

10 Department of Health. Coronary heart disease: national service framework for coronary heart disease: modern standards and service models. London: Department of Health, 2000.

11 Berwick DM, Nolan TW. Physicians as leaders in improving health care. Ann Intern Med 1998; 128:289-92.

12 Carroll J, Edmondson A. Leading organisational learning in health care. Qual Saf Health Care 2002;11:51-6.

13 Lewin K. Frontiers in group dynamics. Hum Relat 1947;1:5-41.

14 Kotter J, Schesinger L. Choosing strategies for change. Harv Bus Rev 1979;57:106-14.

15 Kanter RM. Managing the human side of change. Manage Rev 1985;5:56.

16 Dawson S. Analysing Organisations, 3rd ed. London: Macmillan, 1996.

17 Department of Health. A policy framework for commissioning cancer services: a report by the expert advisory group on cancer to the chief medical officers of England and Wales, (The Calman-Hine Report). London: Department of Health, 1995.

18 Garside P, Black A. Doctors in chambers. Perhaps the time has come for a new way of doing business with consultants. BMJ 2003;326:22. 